\title{
SUR LES PHLEBOTOMES DE L'ÎLE DE' CRÈTE
}

\author{
Par A. RISTORCELLI
}

Dans un lot de 138 phlébotomes envoyés de la Canée (île de Crète) au professeur E. Brumpt (1) par le $\mathrm{D}^{r} \mathrm{E}$. Papantonakis en 1938, nous relevons la présence des quatre espèces suivantes :

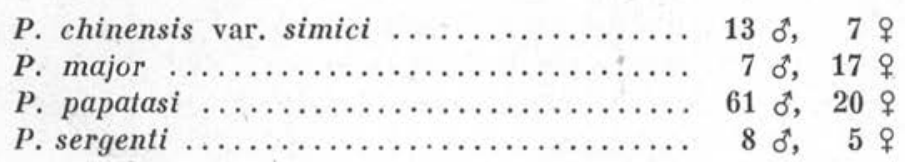

Huit espèces de phlébotomes ont été signalées jusqu'ici dans cette île :

P. chinensis var. simici: par Parrot en 1936; et par Adler, Theodor et Witenberg en 1938.

$P$. major: par les mêmes auteurs.

P. minutus: par Blanc et Langeron en 1922.

P. papatasi : par Blanc et Caminopétros en 1920 ; par Blanc et Langeron en 1922 ; par Parrot en 1936 ; par Adler, Theodor et Witenberg en 1938.

$P$. parroti var. italicus : par Parrot en 1936 ; par Adler, Theodor et Witenberg en 1938.

$P$. perniciosus : par Blanc et Langeron en 1922.

$P$. sergenti: par Blanc et Caminopétros en 1920 ; par Blanc et Langeron en 1922 ; par Parrot en 1936 ; par Adler, Theodor et Witenberg en 1938.

P. vesuvianus : par Parrot en 1936; par Adler, Theodor et Witenberg en 1938.

La proportion des exemplaires pour chacune de nos quatre espèces diffère légèrement des résultats d'Adler, Theodor et Witenberg, dont les recherches portent sur 4.000 échantillons; en parti-

(1) Nous exprimons notre respectueuse gratitude à M. le professeur Brumpt qui a bien voulu nous accueillir et nous confier les riches collections de son laboratoire. Nous remercions également M. le Docteur Parrot de l'Institut Pasteur d'Algérie qui n'a cessé de nous conseiller et qui a déterminé neuf de nos exemplaires.

Annales de Parasitologie, T. XVII, N $4 .-1^{\text {er }}$ juillet 1939 , p. 355-358. 
culier, la proportion que nous obtenons pour le $P$. chinensis var. simici est plus élevée que dans leur étude.

Par ailleurs, nous avons retrouvé, dans les collections du Laboratoire de Parasitologie de la Faculté de médecine de Paris, certains exemplaires récoltés par Blanc et Langeron à Hérakleion en 1922.

L'unique exemplaire mâle de $P$. perniciosus signalé par ces auteurs, à une époque où l'on n'utilisait pas encore certains caractères morphologiques, doit être considéré comme un $P$. major dont il présente en particulier tous les caractères de l'armature génitale : organe intromittent long, à bords presque parallèles et à extrémité hémisphérique.

Un des deux exemplaires mâles d'Hérakleion $\left(\mathrm{n}^{\circ}{ }^{108)}\right.$ de $P$. minutus a été étudié en 1931 par Nitzulescu dans sa révision des phlébotomes du groupe minutus ; l'espèce minutus venait d'être subdivisée (groupe minutus ou sous-genre Prophlebotomus, de Parrot). Nitzulescu rattache l'exemplaire d'Hérakleion à l'espèce $P$. parroti et il donne des figures de l'organe intromittent ainsi que de la cavité buccale.

En 1936, Parrot, dans une note sur les phlébotomes de Crète, pense que l'exemplaire en question doit probablement appartenir à la variété italicus de la même espèce, décrite en 1931 par Adler et Theodor.

Nous avons examiné également cet exemplaire. Les caractères donnés par Nitzulescu de la cavité buccale diffèrent un peu de ceux que nous observons : tandis que Nitzulescu représente près de 32 dents marginales, nous n'en relevons pas plus de 18, par petits groupes de 2 , de 3 ou de 4 ; la plage pigmentée est plus rapprochée de la cavité buccale. Les exemplaires nord-africains de $P$. parroti $\sigma^{\top}$ de la collection du Laboratoire de Parasitologie ont une plage pigmentée presque invisible tant elle est réduite, et une armature buccale composée d'une quinzaine de dents. La zone dentée de l'exemplaire décrit par les auteurs palestiniens renferme 18 dents (exemplaire provenant d'Italie). Dans l'exemplaire d'Hérakleion, toutes les dents ne sont pas sur la même ligne : quelques-unes sont dispersées au-dessus. Comme le constate Nitzulescu, les dents sont disposées comme sur une lame de scie, mais ce serait en réalité une lame de scie ébréchée, où de nombreuses dents seraient absentes. Les dents peuvent être de formes variées : carrées ou aiguës, courtes ou longues, suivant la provenance des exemplaires. Adler et Theodor représentent les dents serrées en peigne ou en palissade et « convergeant vers la ligne médiane ». Une autre 
différence avec la description donnée par Adler et Theodor réside dans la morphologie de l'appáreil génital mâle, l'épine atrophiée de Parrot implantée, d'après ces auteurs, "à peu près au-dessous du milieu du segment », est située sur notre exemplaire au début du dernier tiers du segment distal de la gonapophyse supérieure.

Les mêmes auteurs font reposer la diagnose de leur variété sur le nombre de dents de la cavité buccale de la femelle; la variété italicus serait paucidentée par rapport à l'espèce parroti s. str.; elle ne porterait que 50 dents au lieu de $70-80$; dents plus larges que les dents du parroti s. str. C'est à peu près la seule différence marquée qu'ils signalent. Or, le nombre de dents est essentiellement variable suivant les origines des exemplaires : sur les exem-

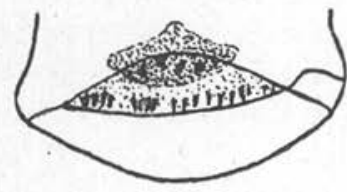

a

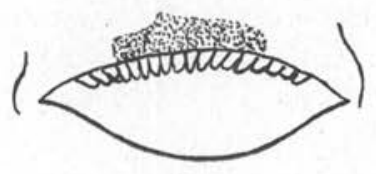

b

Fig. - Armature buccale de $P$. parroti var. italicus mâle: $a$, exemplaire d'Herakleion ; b, exemplaire d'Italie (d'après Adler et Theodor). Au-dessus de la zone dentée on distingue la plage pigmentée.

plaires nord-africains, le nombre de dents est de $34-36$; sur des exemplaires espagnols, de 60-64 ; ce sont ces derniers, d'ailleurs, qui paraissent posséder le plus grand nombre de dents parmi les exemplaires divers que nous avons pu examiner.

Les autres caractères: dimensions, formule palpale, rapport alaire, sont communs aux deux formes.

Les rapports alaires $\frac{\alpha}{\beta}$ sont peu variables ; Adler et Theodor les signalent chez la variété italicus variant de 0,5 à 0,7 . Sur l'exemplaire d'Hérakleion, il est de 0,5 ; sur des exemplaires nord-africains de $P$. parroti s. str., il est de 0,6 .

Les rapports de Raynal $\frac{\text { tibia }}{\text { fémur }}$ sont respectivement pour les exemplaires nord-africains : $1^{\text {re }}$ paire : $0,8-0,9 ; 2^{\circ}$ paire : $1-1,1$; $3^{\circ}$ paire : 1,2-1,3. Sur l'exemplaire d'Hérakleion, les pattes postérieures manquent : pour les $1^{\text {re }}$ et $2^{\circ}$ paires les rapports sont de 0,9 et 1 .

Adler et Theodor, et surtout Nitzulescu, ont fait remarquer la variabilité de la formule palpale dans cette espèce. 
En somme, étant donné le grand nombre de caractères semblables communs au $P$. parroti et au $P$. parroti italicus, et le peu de caractères différentiels fixes (nombre de dents buccales), nous ne pensons pas que la variété italicus doive être maintenue. Même s'il était démontré que les espèces de Phlebotomus parroti de Berbérie soient paucidentées par rapport aux espèces de Phlebotomus parroti du littoral nord-méditerranéen (1) et de Crète, la création de cette variété ne nous semblerait pas devoir s'imposer.

\section{RÉSUMÉ}

Dans cette note, nous rapportons les résultats de la détermination de 138 phlébotomes de l'île de Crète et nous discutons l'existence de la variété italicus du $P$. parroti.

\section{BIBLIOGRAPHIE}

AdLer (S.) and Theodor (O.). - A study of the sandfly population in endemic foci of infantile kala-azar in Italy. Bull. entomol. research, XXII, 1931 , p. 105.

Adeer (S.), Theodor (O.) and Witenberg (G.). - Investigations on mediterranean kala-azar. XI. A study of leishmaniasis in Canea (Crete). Proc. Roy. Soc., B, CXXV, 1938, p. 491.

Blanc (G.). - Liste des insectes piqueurs, etc..., observés en Crète pendant le mois d'août 1922. Arch. Inst. Pasteur Hellénique, I, 1924, p. 239.

Blanc (G.) et Caminopetros (J.). - Enquête sur le bouton d'Orient en Crète. Ann. Inst. Pasteur, XXXV, 1921, p. 151.

Caminopetros (J.). - Sur la faune des phlébotomes de la Grèce. Leur distribution dans les foyers de kala-azar. Bull. Soc. path. exot., XXVII, 1934, p. 450 .

Langeron (M.). - Phlébotomes capturés en Crète. Ann. Parasit., I, 1923, p. 108.

- Mission crétoise de l'Institut Pasteur hellénique. Arch. Inst. Pasteur hellénique, I, 1926, p. 271-292, pl. XIV-XVII.

Nrtzulescu (V.). - Contribution à l'étude des phlébotomes du groupe minutus. Ann. Parasit., IX, 1931, p. 111.

Parrot (L.). - Notes sur les phlébotomes, XIX. - Phlébotomes de Crète. Arch. Inst. Pasteur d'Algérie, XIV, 1936, p. 50.

(1) Comme l'écrivent justement Adler et Theodor, le nouveau minutus, après subdivision de l'espèce minutus, est devenu une espèce africaine, bien que décrit originellement en Italie par Rondani en 1843. Le seul représentant italien du sous-genre Prophlebotomus étant le $P$. parroti dont nous ne reconnaissons pas la variété italicus, il est probable que c'est en réalité le $P$. parroti qui a été vu par Rondani.

Institut de Parasitologie de la Faculté de médecine de Paris (Directeur : Prof. E. Brumpt). 